\title{
Syndrome des spermatozoïdes macrocéphales polyflagelles et Assistance Médicale à la Procréation
}

\author{
Vincent ACHARD, Marie-Roberte GUICHAOUA
}

Laboratoire de Biologie de la Reproduction, AP-HM, Hôpital de la Conception, Marseille

\section{RÉSUMÉ}

Le syndrome des spermatozoïdes macrocéphales est classiquement décrit comme une atteinte de l'ensemble de la population gamétique. L'étiopathogénie de ce syndrome est encore mal déterminée, mais les anomalies méiotiques y sont constantes d'où l'appellation de Déficience de Division Méiotique (Meiotic Division Deficiency, MDD). La ou les mutations en cause ne sont pas déterminées. Plusieurs variants phénotypiques existent.

Nous avons mis en évidence un nouveau variant de ce syndrome, basé sur l'observation de populations gamétiques en mosaïque dans lesquelles coexistent des spermatozoïdes macrocéphales et des spermatozoïdes de taille normale. L'observation de syndromes MDD accompagnés d'une histoire familiale de morts périnatales laisse supposer qu'une atteinte du mécanisme de division des cellules somatiques pourrait accompagner celui des cellules germinales. L'Assistance Médicale à la Procréation (AMP), et plus particulièrement l'injection intra cytoplasmique de spermatozoïde permet d'obtenir des grossesses chez des couples dont le conjoint est porteur de ce syndrome.

Nous envisageons d'étudier des couples dont le conjoint présente une population de spermatozoïdes macrocéphales afin de définir précisément le phénotype morphologique du syndrome des spermatozoïdes macrocéphales en mosaïque (et regrouper les observations en différentes catégories), d'apprécier le type de transmission de ce syndrome par l'établissement d'arbres généalogiques et d'évaluer grâce à la technique d'hybridation in situ en fluorescence le pourcentage de spermatozoïdes de taille normale aneuploïdes. Nous suivrons le parcours de ces couples en AMP et nous rechercherons une incidence accrue de paucifécondation, l'absence d'implantation embryonnaire, de fausses couches spontanées ou de toute anomalie pouvant survenir au cours de la grossesse.

Mots clés : spermatozoïdes macrocéphales, méiose, hybridation in situ en fluorescence, analyse cytogénétique, aberrations chromosomiques

\section{INTRODUCTION - DESCRIPTION DU SYNDROME}

En 1977, l'équipe de Nistal rapporte pour la première fois un cas d'asthénotératospermie associant plusieurs anomalies caractéristiques des spermatozoïdes: une augmentation de taille de la tête avec irrégularité de ses contours, et un nombre de flagelle variable, compris entre 0 et 4 [10]. Par la suite, le regroupement de six cas similaires permet, grâce à l'analyse ultrastructurale quantitative, d'objectiver une multiplication par trois du volume nucléaire et de montrer la présence d'une moyenne de 3,6 axonèmes par tête $[4,6]$. L'étude en microscopie électronique montre également l'existence dans ces spermatozoïdes « macrocéphales " de larges vacuoles nucléaires ainsi que de nombreuses anomalies de l'acrosome $(45 \%$ et $87 \%$ des cas respectivement) [4].

La fréquence des spermatozoïdes anucléés dans ce syndrome ("pinhead spermatozoa ") témoigne de la fragilité de l'insertion du flagelle sur la tête du spermatozoïde [8]. L'analyse cytogénétique du complément chromosomique des spermatozoïdes grâce à l'utilisation de la technique d'hybridation in situ en fluorescence (FISH pour Fluorescent In Situ Hybridization) met en évidence des taux de triploïdies et de tétraploïdies élevés chez les patients atteints $[2,14]$. Cette observation évoque un déficit de division méiotique d'où l'appellation anglo-saxonne de Meiotic Division Deficiency (MDD) [3] (Figure 1).

\footnotetext{
Correspondance :

Dr Vincent ACHARD - laboratoire de Biologie de la Reproduction, AP-HM, Hôpital de la Conception, 147 Boulevard Baille, 13385 Marseille cedex 5. France Tel 04.91.38.13.76 - Fax 04.91.38.38.9 -

Emailvincent.achard@mail.ap-hm.fr,
} 


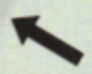

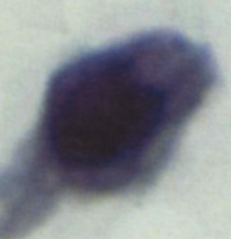

Figure 1 : Spermatozoïde macrocéphale polyflagelle coloré par la coloration de Schorr. Le contour de la tête est irrégulier, avec un acrosome malformé dont l'aspect fait évoquer une duplication. Au niveau du flagelle, trois pièces intermédiaires sont visibles, puis l'un de ces flagelles se duplique au niveau de la pièce principale (flèche). Barre = 10 micromètres.

\section{EVIDENCES PHYSIOPATHOLOGIQUES}

L'analyse histologique testiculaire dans le syndrome MDD montre des cellules en cours de division bloquées en métaphase ainsi que la présence de fuseaux unipolaires [6] ; en outre, un grand nombre de cellules dégénèrent. Les rares éléments de la lignée germinale qui poursuivent leur différenciation présentent une manchette courte, le plus souvent unilatérale, dont la fonction morphogénétique est alors altérée. Cette anomalie peut expliquer la forme irrégulière du noyau observée dans les spermatozoïdes macrocéphales [5]. Des fuseaux de division anormaux unipolaires au cours des métaphases I et II, pouvant être situés soit en périphérie de la cellule, soit en position centrale ont été observés en microscopie électronique [5]. Les chromosomes métaphasiques ont alors une disposition en étoile [5], et la division semble bloquée à ce stade. La duplication des centrio-

les s'opérerait normalement, expliquant la formation de plusieurs flagelles [6]. Des anomalies des kinétochores sur lesquels se fixent les microtubules kinétochoriaux ont également été évoquées pour expliquer des déficits de migration des chromatides sœurs [3].

Des modèles expérimentaux du syndrome MDD existent : $20 \%$ des mutants mâles stériles de l'espèce Drosophila présentent un phénotype germinal comparable à celui observé en pathologie humaine.

Chez la drosophile, des mutations de gènes codant pour des protéines du cytosquelette [9] ou pour des éléments de régulation du cycle cellulaire ont été identifiées. Twine, par exemple, est une phosphatase nécessaire à l'association de la gamma-tubuline Tub23C au centriole au cours de la première division de méiose [13]. Diverses études ont également montré l'importance d'éléments de contrôle post-traductionnels (stabilisation de l'ARN messager) ou posttranscriptionnels (stabilisation de la molécule) dont le meilleur exemple est Boule, une protéine de liaison à l'ARN, exprimée uniquement dans le testicule, et qui régule de manière post-transcriptionnelle twine. L'existence d'anomalies du cytosquelette est confortée par l'effet de drogues dirigées contre les tubulines qui reproduisent le phénotype MDD [12].

Alors que plus d'une centaine de lignées de souris knockout présentent des anomalies de la spermatogenèse associées à une infertilité, il n'existe à l'heure actuelle pas de modèle murin du syndrome MDD [3].

\section{VARIANTS PHÉNOTYPIQUES}

Trois variants phénotypiques du syndrome MDD ont déjà été décrits.

Le type 1 correspond au syndrome tel qu'il a été initialement définit [5]. On y observe une oligospermie d'importance variable selon les cas $\left(0,5 \times 10^{6}\right.$ spermatozoïdes $/ \mathrm{ml}$ [11] $-39,9 \times 106$ [4] spermatozoïdes $/ \mathrm{ml}$ ). On observe également une atteinte de la majorité des spermatozoïdes [4] avec une augmentation de la taille du noyau, une forme atypique de la tête qui présente des extensions apicales, ainsi que la présence de plusieurs flagelles [3].

La description du type 2 est basée sur des observations de biopsies testiculaires dans lesquelles le déficit de division méiotique est associé à un arrêt au stade spermatide ronde, donnant un aspect de spermatide macronucléée. Cette anomalie résulterait selon les auteurs d'une absence complète d'élongation nucléaire, alors qu'elle est partielle dans le type 1 grâce à la manchette unilatérale [7], ainsi que d'un défaut de formation de l'acrosome. Neuf patients porteurs de ce syndrome montraient une azoospermie, seul un présentait une oligospermie ainsi que la présence de nombreuses cellules germinales immatures dans l'éjaculât.

Le type 3 diffère du type 1 par l'existence d'une anomalie de la croissance de l'axonème qui entraîne la formation de structures péri-axonémales désorganisées, avec un flagelle écourté [4]. 


\section{UNE NOUVELLE FORME DU SYNDROME}

Nous avons observé que chez certains patients seul un clone de cellules germinales présente les caractéristiques du syndrome des spermatozoïdes macrocéphales [14]. Ces formes en mosaïque doivent être considérées comme un autre variant du syndrome MDD, elles sont beaucoup plus fréquentes que les autres formes décrites jusqu'à présent. Dans ces formes, des spermatozoïdes macrocéphales réguliers et des spermatozoïdes dupliqués sont associés avec une fréquence variable aux spermatozoïdes macrocéphales irréguliers. La coexistence de ces deux types cellulaires peut résulter soit de la présence d'une mosaïque germinale, soit de l'expression variable et de la pénétrance incomplète d'une mutation, qui présenterait une sensibilité au micro-environnement testiculaire. La technique de FISH réalisée chez deux de nos patients montre que des spermatozoïdes de taille normale peuvent aussi présenter des aneuploïdies chez ces patients avec une incidence supérieure à celle des témoins normaux.

\section{HÉRÉDITÉ ET SYNDROME DES SPERMATOZOIIDES MACROCÉPHALES}

L'hypothèse longtemps suspectée d'une mutation génique fut étayée à deux reprises : la première observation se base sur une série de 17 patients présentant des spermatozoïdes macrocéphales dans l'éjaculât. Plus de la moitié d'entre eux présentaient une histoire familiale d'infertilité masculine. Les patients ne présentaient pas de caractéristique ethnique commune, l'existence d'une consanguinité parentale n'a pas été recherchée, et les caryotypes, réalisés chez 10 patients, étaient normaux [8]. La seconde observation décrit une forme familiale de ce syndrome dans laquelle deux frères infertiles sont porteurs d'une forme homogène. Le caryotype, réalisé sur l'un des deux frères seulement, s'est révélé normal. II n'existe pas de consanguinité entre les parents de ces deux patients [1].

A l'heure actuelle, la (ou les) mutation(s) responsable(s) de ces anomalies des spermatozoïdes n'a ou (n'ont) pas encore été identifiée(s) mais les différentes formes morphologiques observées suggèrent l'existence de formes génétiquement différentes.

\section{ANOMALIE MÉIOTIQUE STRICTE OU ASSOCIATION A UNE ANOMALIE MITOTIQUE ?}

Jusqu'en 2001, il était admis que le syndrome des spermatozoïdes macrocéphales polyflagelles était spécifique de la méiose. L'observation réalisée par l'équipe de Benzacken en 2001 [1] remet en question cette notion : elle décrit pour la première fois un cas familial de syndrome MDD associé à huit morts périnatales dans la fratrie. Ceci soulève l'hypothèse d'une atteinte somatique associée à l'atteinte méiotique.

\section{SYNDROME MDD ET TRAITEMENT DE L'INFERTILITÉ}

L'asthénotératospermie totale présentée dans les formes homogènes du syndrome MDD est incompatible avec une fécondation naturelle. Des injections intra-cytoplasmiques (ICSI) ont été tentées chez ces patients [8], elles ont permis d'obtenir des grossesses, mais avec des taux de fécondation et de grossesses inférieurs à ceux obtenus chez les couples dont l'homme présente une tératospermie polymorphe d'importance équivalente. Ces faibles taux de fécondation et de grossesses résultent probablement de la présen$c e$, chez ces patients, d'un pourcentage élevé d'embryons porteurs de déséquilibres chromosomiques.

La survenue d'embryons au contenu génétique anormal peut être liée soit à la micro-injection d'un spermatozoïde porteur d'un déséquilibre chromosomique, soit à la présence d'une mutation qui s'exprime à la fois dans le tissu germinal et dans le tissu somatique, entraînant ainsi des anomalies de répartition des chromosomes au cours des premières divisions de segmentation de l'embryon. Aucune malformation néonatale n'a été signalée jusqu'à présent mais le nombre de naissance est très faible actuellement ( 3 naissances). La fréquence de ce syndrome dans notre centre a été évaluée à 2,7 pour mille.

\section{OBJECTIFS DE L'ÉTUDE}

Dans le cadre de la bourse de recherche allouée par la Société d'Andrologie de Langue Française, le projet de recherche poursuivra les objectifs suivants :

- Apprécier le type de transmission du syndrome MDD et rechercher les éventuels troubles de la reproduction associés à ce syndrome par l'établissement d'un arbre généalogique.

- Etablir le phénotype précis du syndrome MDD pour chaque patient par l'analyse du spermogramme en évaluant les différents types de spermatozoïdes macrocéphales : irréguliers, réguliers et les spermatozoïdes dupliqués.

- Apprécier, par la technique d'hybridation in situ en fluorescence, l'implication préférentielle de la 1 ère ou de la

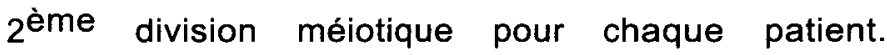
Rechercher une éventuelle corrélation entre l'implication prédominante de la $2^{\text {ème }}$ division et la fréquence des avortements et des morts périnatales. Evaluer pour chaque patient le pourcentage de spermatozoïdes aneuploïdes de taille normale, qui pourraient être micro-injectés dans les ovocytes au cours des ICSI et représenter un facteur supplémentaire de déséquilibre chromosomiques embryonnaires.

- Développer la technique d'immunocytochimie sur les protéines centriolaires des spermatozoïdes MDD et des cellules germinales immatures présentes dans le sperme de ces patients.

- Définir la conduite à tenir lors de la survenue d'une grossesse spontanée ou après ICSI : estimer la nécessité d'un conseil génétique afin d'évaluer la nécessité de réaliser un diagnostic prénatal. 
1. BENZACKEN B., GAVELLE F.M., MARTIN-PONT B. et al. : Familial sperm polyploidy induced by genetic spermatogenesis failure : case report. Hum. Reprod,. 2001, 16 : 2646-2651.

2. DEVILLARD F., METZLER-GUILLEMAIN C., PELLETIER R. et al. : Polyploidy in large-headed sperm : FISH study of three cases. Hum. Reprod., 2002, 17 : 1292-1298.

3. ESCALIER D. : Genetic approach to male meiotic division deficiency : the human macronuclear spermatozoa. Mol. Hum. Reprod., 2002, $8: 1-7$.

4. ESCALIER D. : Human spermatozoa with large heads and multiple flagella : a quantitative ultrastructural study of 6 cases. Biol. Cell, 1983, $48:$ 65-74.

5. ESCALIER D. : Microtubule disorders in human spermatozoa. In : De Brabander $M$. editor. Microtubules and Microtubule Inhibitors. Amsterdam, Elsevier Science, 1985 : 345-352.

6. ESCALIER D. : Thèse de doctorat d'état. Paris : Université Paris XI ; 1985.

7. ESCALIER D., BERMUDEZ D., GALLO J.M., VIELLEFOND A., SCHREVEL J. : Cytoplasmic events in human meiotic arrest as revealed by immunolabelling of spermatocyte proacrosin. Differentiation, 1992, $51: 233-243$.

8. KAHRAMAN S., AKARSU C., CENGIZ G. et al. : Fertility of ejaculated and testicular megalohead spermatozoa with intracytoplasmic sperm injection. Hum. Reprod., 1999, 14 : 726-730.

9. KEMPHUES K.J., KAUFMAN T.C., RAFF R.A., RAFF E.C. : The testis-specific beta-tubulin subunit in Drosophila melanogaster has multiple functions in spermatogenesis. Cell, 1982, $31: 655-670$.

10. NISTAL M., PANIAGUA R., HERRUZO A. : Multi-tailed spermatozoa in a case with asthenospermia and teratospermia. Virchows Arch. B. Cell. Pathol., 1977, 26 : 111-118.

11. TASDEMIR I., TASDEMIR M., TAVUKCUOGLU S., KAHRAMAN S., BIBEROGLU K. : Effect of abnormal sperm head morphology on the outcome of intracytoplasmic sperm injection in humans. Hum. Reprod., 1997, $12: 1214-1217$.

12. WILKINSON R.F., STANLEY H.P., BOWMAN J.T. : Genetic control of spermiogenesis in Drosophila melanogaster : the effects of abnormal cytoplasmic microtubule populations in mutant $\mathrm{ms}(3) 10 \mathrm{R}$ and its colcemid-induced phenocopy. J. Ultrastruct. Res., 1974, $48:$ 242-258.

13. WILSON P.G., ZHENG Y., OAKLEY C.E. et al. : Differential expression of two gamma-tubulin isoforms during gametogenesis and development in Drosophila. Dev. Biol., 1997, 184 : 207-221.

14. YUROV Y.B., SAIAS M.J., VORSANOVA S.G. et al. : Rapid chromosomal analysis of germ-line cells by $\mathrm{FISH}$ : an investigation of an infertile male with large-headed spermatozoa. Mol. Hum. Reprod., 1996, 2 : 665-668.

Bourse SALF 2004 de Recherche en Andrologie.

Manuscrit reçu : mars 2005 ; accepté mars 2005

\section{Macrocephalic spermatozoa syndrome and Assisted Reproductive Medicine}

Vincent ACHARD, Marie-Roberte GUICHAOUA

The first case of macrocephalic spermatozoa with multiple tails was described in 1977 by Nistal et al. In its classical form (type I), the syndrome comprises a combination of oligoasthenoteratozoospermia, sperm heads of increased size with irregular shapes and multiple flagellae. Fluorescent in situ hybridization (FISH) on sperm chromosomes reveals disorders such as diploidy, triploidy, tetraploidy or aneuploidy. A genetic origin is strongly suggested by the existence of familial cases.

Although the pathophysiology has not been fully elucidated, meiotic disorders are constantly found giving this syndrome the name of Meiotic Division Deficiency (MDD). Several cellular structures may be involved: cytoskeleton disorders (spindle or centrosome) could give rise to abnormal chromosome distribution during meiosis and could explain irregular head shapes. Numerous phenotypes have already been described.

We observed that a population of men attending our infertility center showed an incomplete form of the syndrome, with only a clone of macrocephalic germinal cells. This observation is more common than the classical type. FISH on the spermatozoa of these patients show that normalsized spermatozoa can be aneuploid, with a higher incidence than controls.

The description of a family history of MDD with multiple perinatal deaths reveals that a somatic cell disorder can be associated. In the common type, the low sperm count found in MDD is not compatible with natural fertilization. Intracytoplasmic sperm injections (ICSI) have been performed for these patients, giving rise to pregnancies although fertilization and pregnancy rates were low. Embryos with an incorrect set of chromosomes could result from the injection of aneuploid spermatozoa, or could be due to a mutation affecting both germinal and somatic cell lines, thus explaining abnormal chromosome distribution in the very first segmentation divisions of the embryo.

We plan to study couples attending our infertility center in which men show an increased percentage of macrocephalic spermatozoa, in order to precisely define the morphological phenotype of the mosaic macrocephalic spermatozoa syndrome. We will also assess the type of genetic transmission involved and will use FISH to estimate the percentage of normal-sized spermatozoa showing abnormal chromosome numbers. Follow-up of the couple is necessary for evaluation of fertilization and pregnancy rates in this syndrome.

Key-words : macrocephalic spermatozoa, meiosis, fluorescent in situ hybridization, cytogenetic analysis, chromosome abnormalities 